\title{
Synthesis and in vivo Anti-inflammatory and Analgesic activities of Oxadiazoles clubbed with Benzothiazole nucleus
}

\author{
Vishal Kumar1,2, Saurabh Sharma ${ }^{1}$ and ${ }^{*}$ Asif Husain ${ }^{3}$ \\ ${ }^{1}$ Department of Pharmacy, Vivek College of Technical Education Bijnor-246726 (U.P.), India \\ ${ }^{2}$ Uttarakhand Technical University, Post Office Chandanwadi, Prem Nagar, Suddhowala, Dehradun- 248007 (U.K), India \\ ${ }^{3}$ Department of Pharmaceutical Chemistry, Faculty of Pharmacy, Jamia Humdard, New Delhi-110062, India
}

\begin{abstract}
The aim of the present work is to synthesize and evaluate the anti-inflammatory and analgesic activity of 2-(5-substituted-1,3,4oxadiazole-2-yl)-1,3-benzathiazole derivatives. In the present investigation, a series of 2-(5-substituted-1,3,4-oxadiazole-2-yl)-1,3benzathiazole derivatives (3a1-3a9) were synthesized by condensing benzothiazolyl carboxyhydrazide and appropriate aryl acids in the presence of phosphorous oxychloride. Structures of synthesized compounds were established on the basis of IR, ${ }^{1} \mathrm{H}$ NMR, and Mass spectroscopy. All the synthesized compounds were screened for their in-vivo anti-inflammatory and analgesic activities at the dose of $50 \mathrm{mg} / \mathrm{kg}$ and $10 \mathrm{mg} / \mathrm{kg}$ po. The biological result shows that some compounds were good in their anti-inflammatory and analgesic actions.
\end{abstract}

Key Words: 1,3,4-oxadiazole derivatives, synthesis, aryl acids, anti-inflammatory activity, analgesic activity, spectroscopy.

\section{INTRODUCTION}

Benzothiazole is a heterocyclic compound, weak base, having varied biological activities. It is of great scientific interest due to its potential pharmacological and pharmaceutical applications. Benzothiazole and oxadiazole derivatives have been widely researched in bioorganic and medicinal chemistry with applications in drug discovery. Both the nucleuses are well acknowledged to possess a wide range of pharmaceutical, agrochemical, and biological applications. Substituted-oxadiazoles show wide variety of biological activities; anti-inflammatory (Ali et al., 2014) and (Husain and Ajmal, 2009) analgesic (Ali et al., 2010) and (Badiadka et al., 2005) antibacterial (Shridhar et al., Desai et al., 2014, Sahin et al., 2002) antifungal (Oliveira et al., 2013) and anticancer activities (Abu-Zaied et al., 2012 and Valente et al., 2014). Benzothiazole derivatives have also reported to show antiinflammatory (Abbas et al., 2013 and Patil et al., 2015) analgesic (Sharma et al., 2013 and Sharma et al., 2013a) antifungal (Liu et al., 2013) anticancer (Gurdal et al., 2015) and antimicrobial (Sigmundova et al., 2007 and. Singh et al., 2013) activities. Non-steroidal anti-inflammatory drugs NSAIDs configuration a class of therapeutic agents that are most generally used because of their antiinflammatory, analgesic and anti-microbial effects.

In view of these observations, it was considered worthwhile to prepare hybrid derivatives of benzothiazole with oxadiazole with an aim to get potential antiinflammatory and analgesic compounds.

\section{MATERIALS AND METHODS}

\section{Experimental}

${ }^{1} \mathrm{H} \mathrm{NMR}\left(\mathrm{DMSO} / \mathrm{CDCl}_{3}\right.$ ) was taken on Varian E-360 MHz (Perkin-Elmer, USA) or and Brucker spectrometer DPX$300 \mathrm{MHz}$ (Bruker, Germany). Mass Spectra was taken on

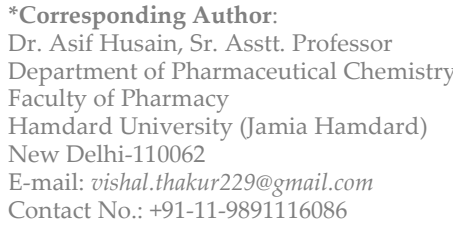

LCMS Shimadzu Spectrophotometer. From open capillary tubes the melting point was determined and all are uncorrected. All the compounds have presented satisfactory chemical analysis. All solvents were distilled previous to use.

General Method for the synthesis of Ethyl-2benzothiazole carboxylate (I)

A mixture of o-aminothiophenol $(0.1 \mathrm{~mol})$ and diethyl oxalate $(0.2 \mathrm{~mol})$ upon heating on reflux for 5 hours, during experiment the temperature was decreased from 146 to $92^{\circ} \mathrm{C}$. After cooling, the mixture was added into a solution of $50 \mathrm{ml}$ of conc. $\mathrm{HCl}, 150 \mathrm{ml}$ of water and $70 \mathrm{ml}$ of ethanol with stirring, the oil dissolved and a solid formed. The mixture was cooled; the product was filtered and washed with aq. ethanol then dried and finally recrystallized from pet ether.

General Method for the synthesis of 1, 3-benzothiazole2-carboxyhydrazide (II)

In a dry $100 \mathrm{ml}$ round bottomed flask (RBF) the ethyl-2benzothiazole carboxylate (I) ( 0.01 mole) was dissolved in ethanol $(60 \mathrm{ml})$. The hydrazine hydrate $(0.02 \mathrm{~mole})(99 \%)$ was added drop by drop with constant stirring and the contents were refluxed for $8 \mathrm{hrs}$, cooled at room temperature. The solid separated was filtered and washed with water and dried, and finally recrystallized from ethanol.

General Method for the synthesis of 2-(5-substituted1,3,4-oxadiazole-2-yl)-1,3-benzothiazole (3a1-a9)

A mixture of 1,3-benzothiazole-2-carboxyhydrazide (II) $(0.01 \mathrm{~mol})$, suitable aromatic acid $(0.02 \mathrm{~mol})$ and phosphoryl chloride $(10 \mathrm{ml})$ was refluxed on steam bath for 5-6 hours. After standing at room temperature it was added into the crushed ice with continuous stirring. The solid crystals obtained, were filtered, washed with water and recrystallized from ethanol to obtain final compound (3a13a9). The physicochemical parameters of these compounds are presented in table 1 and synthesis pathway of resulted compounds is given in Scheme 1. 
<smiles>Nc1ccccc1S</smiles><smiles>CCOC(=O)C(=O)OCC</smiles>

o-Amino thiophenol

Diethyl oxalate<smiles>CCOC(=O)c1nc2ccccc2s1</smiles>

(1)<smiles></smiles>

(2)<smiles></smiles>

$\left(3 a_{1}-3 a_{9}\right)$

Scheme 1: Synthesis pathway of all resultant compounds (3a1-3a9).

\section{Pharmacology}

The animals used in the examination were sheltered in congruence of the Vivek College of Technical education Unit, which follows the guidelines and regulation set by the committee for the control and administration of experiments on animals (CPCSEA), Ministry of social justice and empowerment, Government of India. The studies were attempted with previous approval from the Institutional Animal Ethics committee (IAEC) and ultimate care was taken to establish that the animals were handling in the most kind and satisfactory manner. Wistar rats and albino mice of either sex (Vivek College of Technical education, India), weighing 150-200 gm and 20$25 \mathrm{gm}$, respectively, were used. Pregnant females were eliminated.

\section{Anti-inflammatory Activity}

Anti-inflammatory activity was carried on the Wistar rats (Winter et al., 1968). The animals were divided into 6 groups each having six animals. Albino rats of either sex weighing 150-200 gm were selected. They were maintained on standard pellet diet and free access to water.<smiles>Cc1ccccc1N</smiles><smiles>Cc1ccc([N+](=O)[O-])cc1</smiles>

$\left(3 \mathrm{a}_{2}\right)$<smiles>Cc1ccccc1O</smiles>

$\left(3 a_{3}\right)$<smiles>Cc1ccc(O)c(O)c1</smiles>

$\left(3 \mathrm{a}_{4}\right)$<smiles>Cc1ccc(N)cc1</smiles>

$\left(3 \mathrm{a}_{5}\right)$<smiles>COc1ccc(C)cc1OC</smiles>

$\left(3 a_{7}\right)$<smiles>Cc1ccc(I)cc1</smiles><smiles>Cc1ccc(N)c(N)c1</smiles>

$\left(3 \mathrm{a}_{8}\right)$<smiles>CCc1cccc2ccccc12</smiles>

$\left(3 a_{9}\right)$

The normal control, Diclofenac and test compounds were administered to the rats 30 minutes before the injection of $0.1 \mathrm{ml}$ of $1 \%$ carrageenan suspension in normal saline. Carrageenan suspension was injected into the sub-planar region of the left hind paw, and the right hind paw served as reference. Immediately thereafter the oedema volume of the injected paws was measured plethysmographically. Anti-inflammatory activity was carried out by (Roy et al., 1980) mercury displacement method.

\section{Analgesic Activity}

The compounds that display good anti-inflammatory active $(>50 \%)$ were protected for analgesic activity. Analgesic activity was carried out by Eddy's hot plate mathod (Kulkarni, 1999). Six groups of albino mice of either sex each comprising of four animals, weighing between 20-25 gms were deprived of food and water for $18 \mathrm{hrs}$ prior to the experiment. The animal with a basal reaction time of less than 8 seconds were considered for the study. The hot plate was stabilized at $55 \pm 1^{\circ} \mathrm{C}$, the animals are placed on the hot plate and the time until either licking or jumping response with the animals is 
Table 1: Characterization of the synthesized compounds.

\begin{tabular}{|c|c|c|c|c|c|c|c|}
\hline \multirow{2}{*}{$\begin{array}{l}\text { Compound } \\
\text { number }\end{array}$} & \multirow[b]{2}{*}{$\mathbf{R}$} & \multirow[b]{2}{*}{$\begin{array}{l}\text { M.p. } \\
\left({ }^{\circ} \mathrm{C}\right)\end{array}$} & \multirow[b]{2}{*}{$\begin{array}{l}\text { Yield } \\
(\%)\end{array}$} & \multirow{2}{*}{$\begin{array}{l}\text { Mol Formula/ } \\
\text { Mol. Mass }\end{array}$} & \multicolumn{3}{|c|}{ Spectral Data } \\
\hline & & & & & $\begin{array}{l}\text { IR (KBr) } \\
(\mathrm{v}, \mathrm{cm}-1)\end{array}$ & $\begin{array}{c}{ }^{1} \mathrm{H} \text { NMR } \\
\left(\mathrm{CDCl}_{3}, \mathrm{DMSO}, \delta, \mathrm{ppm}\right)\end{array}$ & $\begin{array}{l}\text { Mass Spectra } \\
(\mathrm{m} / \mathbf{z})\end{array}$ \\
\hline $3 a 1$ & & $160-162$ & 69 & $\begin{array}{l}\mathrm{C}_{15} \mathrm{H}_{10} \mathrm{~N}_{4} \mathrm{OS} \\
(294)\end{array}$ & $\begin{array}{l}3055(\mathrm{C}-\mathrm{H}) \\
3310(\mathrm{~N}-\mathrm{H})\end{array}$ & $\begin{array}{l}\text { 6.12-7.23 (m, 8H, Ar-H) } \\
\quad 3.93\left(\mathrm{~s}, 2 \mathrm{H},-\mathrm{NH}_{2}\right)\end{array}$ & $294(\mathrm{M}+)$ \\
\hline $3 \mathrm{a} 2$ & & $240-242$ & 65 & $\begin{array}{l}\mathrm{C}_{15} \mathrm{H}_{8} \mathrm{~N}_{4} \mathrm{O}_{3} \mathrm{~S} \\
\quad(324)\end{array}$ & $\begin{array}{l}3025(\mathrm{C}-\mathrm{H}) \\
1315\left(-\mathrm{NO}_{2}\right)\end{array}$ & 6.23-8.11 (m, 8H, Ar-H) & $\mathrm{nr}$ \\
\hline $3 a 3$ & & $242-244$ & 63 & $\begin{array}{l}\mathrm{C}_{15} \mathrm{H}_{9} \mathrm{~N}_{3} \mathrm{O}_{2} \mathrm{~S} \\
\quad(295)\end{array}$ & $\begin{array}{l}3010(\mathrm{C}-\mathrm{H}) \\
3100(-\mathrm{OH})\end{array}$ & $\begin{array}{c}\text { 6.12-7.81 (m, 8H, Ar-H), } \\
9.27(\mathrm{~s}, 1 \mathrm{H},-\mathrm{OH})\end{array}$ & $295(\mathrm{M}+)$ \\
\hline $3 a 4$ & & $170-174$ & 74 & $\begin{array}{c}\mathrm{C}_{15} \mathrm{H}_{9} \mathrm{~N}_{3} \mathrm{O}_{3} \mathrm{~S} \\
(311)\end{array}$ & $\begin{array}{l}3020(\mathrm{C}-\mathrm{H}) \\
3210(-\mathrm{OH})\end{array}$ & $\begin{array}{c}\text { 6.93-7.46 (m, 7H, Ar-H), } \\
9.28(\mathrm{~s}, 2 \mathrm{H},-\mathrm{OH})\end{array}$ & $\mathrm{nr}$ \\
\hline $3 a 5$ & & $210-212$ & 71 & $\begin{array}{l}\mathrm{C}_{15} \mathrm{H}_{10} \mathrm{~N}_{4} \mathrm{OS} \\
\quad(294)\end{array}$ & $\begin{array}{l}3055(\mathrm{C}-\mathrm{H}) \\
3410\left(-\mathrm{NH}_{2}\right)\end{array}$ & $\begin{array}{c}\text { 6.31-7.58 (m, Ar-8H, Ar-H) } \\
3.94\left(\mathrm{~s}, 1 \mathrm{H},-\mathrm{NH}_{2}\right)\end{array}$ & $294(\mathrm{M}+)$ \\
\hline $3 a 6$ & & $180-182$ & 70 & $\begin{array}{l}\mathrm{C}_{15} \mathrm{H}_{8} \mathrm{IN}_{3} \mathrm{OS} \\
\quad(405)\end{array}$ & $\begin{array}{l}3045(\mathrm{C}-\mathrm{H}) \\
1325(-\mathrm{I})\end{array}$ & 6.11-7.64 (m, Ar-8H, Ar-H) & $\mathrm{nr}$ \\
\hline $3 a 7$ & & $178-180$ & 67 & $\begin{array}{c}\mathrm{C}_{17} \mathrm{H}_{13} \mathrm{~N}_{3} \mathrm{O}_{3} \mathrm{~S} \\
(339)\end{array}$ & $\begin{array}{c}3110(\mathrm{C}-\mathrm{H}) \\
2850(\mathrm{C}-\mathrm{H}, \mathrm{ali})\end{array}$ & $\begin{array}{c}\text { 6.24-7.35 (m, 7H, Ar-H), } \\
1.28-2.0835\left(\mathrm{~m}, 6 \mathrm{H},-\mathrm{CH}_{3}\right)\end{array}$ & $\mathrm{nr}$ \\
\hline $3 a 8$ & & $100-104$ & 72 & $\begin{array}{l}\mathrm{C}_{15} \mathrm{H}_{11} \mathrm{~N}_{5} \mathrm{OS} \\
\quad(309)\end{array}$ & $\begin{array}{l}3130(\mathrm{C}-\mathrm{H}) \\
3490\left(-\mathrm{NH}_{2}\right)\end{array}$ & $\begin{array}{l}\text { 6.81-7.94 (m, 7H, Ar-H), } \\
\text { 3.84-4.26 (m, 4H, -NH2) }\end{array}$ & $309(\mathrm{M}+)$ \\
\hline $3 a 9$ & & $106-110$ & 51 & $\begin{array}{c}\mathrm{C}_{20} \mathrm{H}_{13} \mathrm{~N}_{3} \mathrm{OS} \\
(343)\end{array}$ & $\begin{array}{c}3052(\mathrm{C}-\mathrm{H}) \\
2872(\mathrm{C}-\mathrm{H}, \mathrm{ali})\end{array}$ & $\begin{array}{c}\text { 6.13-7.78 (m, 11H, Ar-H), } \\
1.84\left(\mathrm{~s}, 2 \mathrm{H},-\mathrm{CH}_{2}\right)\end{array}$ & $\mathrm{nr}$ \\
\hline
\end{tabular}

$\mathrm{S}$ - singlet, $\mathrm{d}$ - doublet, $\mathrm{t}$ - triplet, $\mathrm{q}$ - quarter, $\mathrm{m}$ - multiplet, $\mathrm{nr}$ - not recorded

recorded by a stopwatch, the time taken as the end point. The reaction time was recorded at prefixed time interval i.e., $0,20,40,60,80,100$ and 120 minutes following oral or subcutaneous administration of the standard or the test compound.

\section{Statistical analysis}

The animal experimental data were indicated as mean \pm SEM. Statistical characteristic between the treatments and the standard were approved by one-way ANOVA pursue by Dunnett's multiple comparison test.

\section{RESULTS AND DISCUSSION}

The synthetic compounds (3a1 - 3a9) were designed for the benzothiazole analogues and has been accomplished starting from the Ethyl-2-benzothiazole carboxylate (I) which was dissolve in ethanol, hydrazine hydrate was added drop by drop with constant stirring and the contents were refluxed for $8 \mathrm{hrs}$, cooled at room temperature. The solid of 1,3-benzothiazole-2-carboxyhydrazide (II) was separated. To it suitable aromatic acid and phosphoryl chloride $(10 \mathrm{ml})$ was added and refluxed on steam bath for 5-6 hours. After standing at room temperature it was added into the crushed ice with continuous stirring. The solid crystals obtained, were filtered, washed with water and recrystallized from ethanol to obtain final compounds (3a1-3a9).

All final compounds were pure and well balanced. The structure elucidation was proved by IR, NMR and Mass spectroscopy. IR spectral peaks of the final compounds were accepted from 3375-3200 $\mathrm{cm}^{-1}$ for $\mathrm{N}-\mathrm{H}$ stretching, $3000-3200 \mathrm{~cm}^{-1}$ for $\mathrm{NH}_{2}, 3075-2850 \mathrm{~cm}^{-1}$ for $\mathrm{C}-\mathrm{H}$ aliphatic and aromatic, $3200-3325 \mathrm{~cm}^{-1}$ for $-\mathrm{OH}$ and 1300 $1400 \mathrm{~cm}^{-1}$ for $\mathrm{NO}_{2}$, respectively (table 1). In ${ }^{1} \mathrm{HNMR}$ spectra typical proton signals for $\mathrm{C}-\mathrm{H}$ aliphatic and aromatic were found near 2.36-3.68, 8.06-6.30 $\delta$ ppm. Mass spectra chanced correct molecular ion peaks.

Compounds (3a1-3a9) were assessing for their in vivo anti-inflammatory activity by the carrageen an induced rat paw edema method. The compounds were assessing at an oral dose $200 \mathrm{mg} / \mathrm{kg}$ and differentiate with the standard drug Diclofenac Sodium. All the compounds showed good anti-inflammatory activities were tested for analgesic activity. All compounds were assessing at 10 $\mathrm{mg} / \mathrm{kg}$ dose as a standard drug Pentazocin. Examination of the results showed that the compound having 1, 3benzothiazole-2-carboxyhydrazide substitution at the $5^{\text {th }}$ position of oxadiazole ring display outstanding analgesic activity (table 2 ). 
Table 2: Data showing anti-inflammatory activity of benzothiazole derivatives (3a1-a9) in (Carrageenan induced acute rat paw oedema model).

\begin{tabular}{|c|c|c|c|c|c|c|c|c|c|c|}
\hline \multirow{3}{*}{ Gp. } & \multirow{3}{*}{ Treatment } & \multirow{3}{*}{$\begin{array}{l}\text { Dose } \\
\mathrm{mg} / \mathrm{kg}\end{array}$} & \multicolumn{8}{|c|}{ Paw oedema volume } \\
\hline & & & \multicolumn{2}{|c|}{ After 1st hour } & \multicolumn{2}{|c|}{ After 2nd hour } & \multicolumn{2}{|c|}{ After 3rd hour } & \multicolumn{2}{|c|}{ After 4th hour } \\
\hline & & & Mean/ \pm SEM & $\%$ ROV & Mean/ \pm SEM & $\%$ ROV & Mean/₫SEM & $\%$ ROV & Mean/ \pm SEM & $\%$ ROV \\
\hline 1 & Control & $1.5 \mathrm{ml}$ & $0.70 \pm 0.025$ & - & $0.76 \pm 0.006$ & - & $0.61 \pm 0.026$ & - & $0.84 \pm 0.015$ & - \\
\hline 2 & Standard & 50 & $0.25 \pm 0.018$ & 64.28 & $0.20 \pm 0.009$ & 73.60 & $0.15 \pm 0.009$ & 74.09 & $0.15 \pm 0.014$ & 82.14 \\
\hline 3 & $3 a 1$ & 200 & $0.25 \pm 0.023$ & 64.28 & $0.21 \pm 0.009$ & 72.36 & $0.15 \pm 0.010$ & 75.40 & $0.20 \pm 0.010$ & 76.19 \\
\hline 4 & $3 \mathrm{a} 2$ & 200 & $0.30 \pm 0.014$ & 57.14 & $0.25 \pm 0.015$ & 67.10 & $0.17 \pm 0.009$ & 71.31 & $0.15 \pm 0.010$ & 81.91 \\
\hline 5 & $3 a 3$ & 200 & $0.31 \pm 0.012$ & 55.71 & $0.29 \pm 0.012$ & 61.84 & $0.23 \pm 0.009$ & 62.29 & $0.25 \pm 0.023$ & 69.28 \\
\hline 6 & $3 a 4$ & 200 & $0.30 \pm 0.020$ & 56.00 & $0.25 \pm 0.023$ & 66.05 & $0.20 \pm 0.012$ & 67.20 & $0.16 \pm 0.014$ & 80.23 \\
\hline 7 & $3 a 5$ & 200 & $0.33 \pm 0.009$ & 52.42 & $0.28 \pm 0.018$ & 62.76 & $0.22 \pm 0.009$ & 63.11 & $0.17 \pm 0.014$ & 79.16 \\
\hline 8 & $3 a 6$ & 200 & $0.30 \pm 0.023$ & 56.00 & $0.25 \pm 0.022$ & 66.05 & $0.20 \pm 0.007$ & 67.20 & $0.16 \pm 0.007$ & 80.23 \\
\hline 9 & $3 a 7$ & 200 & $0.31 \pm 0.016$ & 55.71 & $0.28 \pm 0.011$ & 62.72 & $0.21 \pm 0.009$ & 66.22 & $0.23 \pm 0.009$ & 70.50 \\
\hline 10 & $3 a 8$ & 200 & $0.30 \pm 0.020$ & 57.14 & $0.25 \pm 0.018$ & 67.10 & $0.16 \pm 0.020$ & 73.32 & $0.15 \pm 0.009$ & 81.54 \\
\hline 11 & $3 a 9$ & 200 & $0.32 \pm 0.018$ & 51.12 & $0.28 \pm 0.023$ & 61.70 & $0.23 \pm 0.009$ & 66.80 & $0.18 \pm 0.014$ & 79.08 \\
\hline
\end{tabular}

Animals: Albino rat, Route: P.O, Dose: $20,25 \mathrm{mg} / \mathrm{kg}$ for anti-inflammatory activity. Mean \pm SEM, $\mathrm{n}=4$

Table 3: Data showing analgesic activity of benzothiazole derivatives (3a1-3a9).

\begin{tabular}{|c|c|c|c|c|c|c|c|c|c|c|}
\hline \multirow{2}{*}{ Treatment } & \multirow{2}{*}{$\begin{array}{l}\begin{array}{l}\text { No. of } \\
\text { mice }\end{array} \\
\text { ming }\end{array}$} & \multirow{2}{*}{$\begin{array}{l}\text { Avg. } \\
\text { wt. (g) }\end{array}$} & \multirow{2}{*}{$\begin{array}{c}\text { Average } \\
\text { Dose (mg) }\end{array}$} & \multicolumn{7}{|c|}{ Basal reaction time (sec.) after } \\
\hline & & & & 0 min & $20 \mathrm{~min}$ & $40 \mathrm{~min}$ & $60 \mathrm{~min}$ & $80 \mathrm{~min}$ & $100 \mathrm{~min}$ & $120 \mathrm{~min}$ \\
\hline $\begin{array}{c}\text { Control } \\
\text { (gum acacia) }\end{array}$ & 4 & 22 & - & $3.9 \pm 0.367$ & $4.12 \pm 0.473$ & $4.12 \pm 0.553$ & $4.22 \pm 0.503$ & $4.27 \pm 0.324$ & $3.88 \pm 0.372$ & $5.20 \pm 0.647$ \\
\hline $\begin{array}{l}\text { Standard(Penta- } \\
\text { zocin }(10 \mathrm{mg} / \mathrm{kg})\end{array}$ & 4 & 23.6 & 0.236 & $6.53 \pm 0.889$ & $12.87 \pm 1.332$ & $12.91 \pm 1.320$ & $13.87 \pm 0.279$ & $13.65 \pm 0.851$ & $11.83 \pm 1.042$ & $12.33 \pm 0.918$ \\
\hline Compound(3a1) & 4 & 24.33 & 2.433 & $3.36 \pm 0.521$ & $4.2 \pm 0.351$ & $10.16 \pm 1.837$ & $9.53 \pm 1.080$ & $12.273 \pm 1.093$ & $11.326 \pm 1.374$ & $11.18 \pm 1.955$ \\
\hline Compound(3a2) & 4 & 22.33 & 2.233 & $3.433 \pm 0.212$ & $13.373 \pm 0.521$ & $13.073 \pm 1.219$ & $11.866 \pm 1.611$ & $14.493 \pm 0.320$ & $13.24 \pm 1.111$ & $10.39 \pm 0.610$ \\
\hline Compound(3a3) & 4 & 2 & 2.5 & $4.093 \pm 0.239$ & $4.26 \pm 0.130$ & $12.976 \pm 0.645$ & $12.243 \pm 0.850$ & $11.136 \pm 1.145$ & $10.116 \pm 1.520$ & $9.146 \pm 0.938$ \\
\hline Compound(3a4) & 4 & 26 & 2.6 & $4.116 \pm 0.748$ & $12.47 \pm 1.600$ & $11.903 \pm 1.498$ & $13.23 \pm 0.202$ & $12.446 \pm 1.176$ & $10.986 \pm 0.516$ & $11.663 \pm 0.757$ \\
\hline Compound(3a5) & 4 & 20.66 & 2.066 & $5.156 \pm 0.299$ & $7.106 \pm 0.431$ & $12.203 \pm 0.866$ & $14.55 \pm 0.284$ & $12.991 \pm 0.938$ & $14.793 \pm 0.130$ & $13.2 \pm 1.138$ \\
\hline Compound(3a6) & 4 & 23.33 & 2.333 & $3.836 \pm 0.202$ & $6.066 \pm 0.368$ & $7.31 \pm 0.719$ & $10.59 \pm 0.628$ & $12.76 \pm 0.774$ & $14.04 \pm 0.450$ & $13.42 \pm 0.912$ \\
\hline Compound(3a7) & 4 & 24.33 & 2.433 & $4.977 \pm 0.540$ & $5.497 \pm 0.417$ & $10.990 \pm 1.075$ & $13.063 \pm 0.897$ & $13.607 \pm 0.881$ & $13.693 \pm 0.826$ & $12.093 \pm 1.143$ \\
\hline Compound(3a8) & 4 & 24.33 & 2.433 & $3.36 \pm 0.521$ & $4.2 \pm 0.351$ & $10.16 \pm 1.837$ & $9.53 \pm 1.080$ & $12.273 \pm 1.093$ & $11.326 \pm 1.374$ & $11.18 \pm 1.955$ \\
\hline Compound(3a9) & 4 & 26 & 2.6 & $4.116 \pm 0.748$ & $12.47 \pm 1.600$ & $11.903 \pm 1.498$ & $13.23 \pm 0.202$ & $12.446 \pm 1.176$ & $10.986 \pm 0.516$ & $11.663 \pm 0.757$ \\
\hline
\end{tabular}

Dose: $20,25 \mathrm{mg} / \mathrm{kg}$ for analgesic activity. Mean \pm SEM, $\mathrm{n}=4$

\section{Biological Studies}

From the literature survey it reveals that novel benzothiazole have been reported for number of pharmacological activities and some molecules have shown significant activities and some compounds shows moderate and good activities. Here we have synthesized some novel benzothiazole analogues and screened them for their antiinflammatory and analgesic activities. All compounds at dose of $200 \mathrm{mg} / \mathrm{kg}$ exhibited significant antiinflammatory activity in acute inflammatory models in rats. Results are tabulated in table 3 . Compounds $3 a_{1}, 3 a_{2}$, 3a3, За4, 3a5, 3a6, 3a7, 3a8, and 3a9 exhibited maximum inhibition with $76.19 \%, 81.91 \%, 69.28 \%, 80.23 \%, 79.16 \%$, $80.23 \%, 70.50 \%, 81.54 \%$ and $79.08 \%$, respectively, whereas standard Diclofenac Sodium showed reduction in oedema volume by $82.14 \%$ in carrageenan induced rat hind paw oedema model. The synthesized compounds have shown a significant Analgesic activity. Results are tabulated in table 3 . The compounds $3 a_{5}$ and $3 a 7$ have shown potent Analgesic activity. The compounds 3a3, 3a4, 3a9 showed a moderate analgesic activity. The other compound $3 \mathrm{a}_{1}$ and $3 \mathrm{a}_{2}$ and $3 \mathrm{a}_{6}$ also showed a significant analgesic activity till 120 minutes.

\section{CONCLUSION}

From the above result it has been concluded that a series of nine compounds were prepared by reacting substituted Ethyl-2-benzothiazole carboxylate and 3-benzothiazole-2carboxyhydrazide to form 2-(5-substituted-1,3,4-oxadiazole-2-yl)-1,3-benzothiazole derivatives. Adopting easy and useful method and characterized by TLC, M.P and spectral analysis. Synthesized compounds were screened for their in vivo anti-inflammatory and Analgesic activities. Compounds 3a2, 3a4, 3a6, 3as, showed potent antiinflammatory activity and the compounds 3a5, 3a7 has shown potent Analgesic activity. 


\section{REFERENCES}

Abbas, EMH., Amin, KM., El-Hamouly, WS., Dawood, DH., Abdalla, MM., (2013). Synthesis, anti-inflammatory and antinociceptive activity of some novel benzothiazole derivatives, Research on chemical Intermediates, 41(4),2537-2555.b [DOI]

Abu-Zaied, MAZ., Nawwar, GAM., Swellem, RH., El-Sayed, SH., (2012). Synthesis and Screening of New 5-Substituted-1,3,4-oxadiazole-2thioglycosides as Potent Anticancer Agents, Pharmacology \& Pharmacy, 3(2), 254-261. [DOI]

Ali, A., Mousavi, Z., Tajik, M., Assarzadeh, MJ., Shafiee, A., (2014) Synthesis, analgesic and anti-inflammatory activities of new methylimidazolyl-1,3,4-oxadiazoles and 1,2,4-triazoles, Daru, 22 (1), 22. [DOI]

Ali, A., Shafiee, A., Abdollahi, M., Noeparast, A., Shahrokhinejad, N., Vousooghi, N., Tabatabai, SA., Khorasani, R., (2010). Synthesis and analgesic activity of new 1,3,4-oxadiazoles and 1,2,4-triazoles, Medicinal Chemistry Research, 20(4), 435-442. [DOI]

Desai, NC., Dodiya, AM., Rajpara, KM., Rupala, YM., (2014). Synthesis and antimicrobial screening of 1,3,4-oxadiazole and clubbed thiophene derivatives, Journal of Saudi Chemical Society, 18(3), 255-261. [DOI]

Gurdal, E., Buclulgan, E., Durmaz, I., Cetin-Atalay, R., Yarim, M (2015). Synthesis and Anticancer Activity Evaluation of Some BenzothiazolePiperazine Derivatives, Anti-Cancer Agents in Medicinal Chemistry 1, 15( 3), 382 -389. [DOI]

Husain, A and Ajmal, M., (2009). Synthesis of novel 1,3,4-oxadiazole derivatives and their biological properties, Acta Pharmaceutica, 59(2), 223-233. [DOI]

Kulkarni, S.K., (1999). Hand book of Experimental Pharmacology, Vallabh Prakashan, New Delhi. ISBN 978-3-642-25950-0.

Liu, Y., Wang, Y., Dong, G., Zhang, Y., Wu, S., Miao, Z., Yao, J., Zhang, W. Sheng, C., (2013). Novel benzothiazole derivatives with a broad antifungal spectrum: design, synthesis and structure-activity relationships, Med. Chem. Commun, 4(12), 1551-1561. [DOI]

Narayana, B., Raj, KKV., Valamaleshalatha, B., Kumari, NS., (2005). Synthesis of Some New 2-(6-Methoxy-2-Naphthyl)-5-Aryl-!,3,4Oxadiazoles as Possible Non-steroidal Anti-inflammatory and Analgesic Agents, Archiv der Pharmazie, 338(8), 373-377. [DOI]

Oliveira, CS., Lira, BF., Barbosa-Filho, JM., Lorenzo, JGF., Menezes, CP. Jessyca, M G., Lima, EO., Athayde-Filho, PF., (2013). Synthesis and testing of 3-acetyl-2,5-disubstituted-2,3-dihydro-1,3,4- oxadiazole derivatives forantifungal activity against selected Candida Species, Journal of the Brazilian Chemical Society, 24(1), 115-120. [DO]
Patil, V, Asrondkar, A, Bhadane, V., Bobade, AS, Chowdhary, A., (2015). Synthesis and Anti-inflammatory activity of 2-amino-6-methoxy benzothiazole derivative, Journal of Applied Chemistry, 8(1), 1-2.

Roy A., Roy SM., Gupta J K., and Lahari SC., (1980). Further Studies on anti-inflammatory activity of two potent indian-1- acetic acids. Indian J Physiol Pharmacol, 24, 369. ISSN:0019-5499

Sahin, G., Palaska, E., Ekizoğlu, M., Ozalp, M., (2002). Synthesis and antimicrobial activity of some 1,3,4-oxadiazole derivatives, Farmaco, 57(7), 539-42. [DOI]

Sharma P C., Kumar R., Chaudhary M., Sharma A., Rajak H., (2013). Synthesis and biological evaluation of novel benzothiazole clubbed fluoroquinolone derivatives, J Enzyme Inhib Med Chem, 28(1), 1-10. [DOI]

Sharma, PC., Kumar, R., Chaudhary, M., Sharma, A., Rajak, H., (2013)a Synthesis and biological evaluation of novel benzothiazole clubbed fluoroquinolone derivatives, J Enzyme Inhib Med Chem, 10 (28), 1-10.

Shridhar, A., (2011). Synthesis of Some Novel Bis 1,3,4-Oxadiazole Fused Azo Dye Derivatives as Potent Antimicrobial Agents, International Research Journal of Pure and Applied Chemistry, 1(3), 119 - 129. [DOI]

Sigmundová, IZ., Magdolen, P., Bujdáková, H., (2007). Synthesis and study of new antimicrobial benzothiazoles substituted on heterocyclic ring, ARKIVOC, 8, 183-192. [DOI]

Singh, MK., Tilak, R., Nath, G., Awasthi, SK., Agarwal, A., (2013). Design, synthesis and antimicrobial activity of novel benzothiazole analogs, European Journal of Medicinal Chemistry, 63, 635-644. [DOI]

Valente, S., Trisciuoglio, D., De Luca, T., Nebbioso, A., Labella, D., Lenoci, A., Bigogno, C., Dondio, G., Miceli, M., Brosch, G., Bufalo, DD., Altucci, L., Mai, A., (2014). 1,3,4-Oxadiazole-containing histone deacetylase inhibitors: anticancer activities in cancer cells, J Med Chem, 57(14), 25965. [DOI]

Winter, CA., Risley, EA., Nuss, GW., (1968). Carrageenin-induced edema in hind paw of the rat as an assay for anti-inflammatory drugs, Proceedings of the Society for Experimental. Biology and Medicine, 111,544-547. [DOI] 